\title{
Strongly Extreme Points and Middle Point Locally Uniformly Convex in Orlicz Spaces Equipped with s-Norm
}

\author{
Yunan Cui and Yujia Zhan $\mathbb{D}$ \\ Department of Mathematics, Harbin University of Science and Technology, Harbin 150080, China \\ Correspondence should be addressed to Yujia Zhan; 1051065620@qq.com
}

Received 27 December 2018; Accepted 3 February 2019; Published 3 March 2019

Academic Editor: Miguel Martin

Copyright (C) 2019 Yunan Cui and Yujia Zhan. This is an open access article distributed under the Creative Commons Attribution License, which permits unrestricted use, distribution, and reproduction in any medium, provided the original work is properly cited.

As is well known, the extreme points and strongly extreme points play important roles in Banach spaces. In this paper, the criterion for strongly extreme points in Orlicz spaces equipped with s-norm is given. We complete solved criterion-Orlicz space that generated by Orlicz function. And the sufficient and necessary conditions for middle point locally uniformly convex in Orlicz spaces equipped with s-norm are obtained.

\section{Introduction}

The extreme point set plays a crucial role in function analysis, convex analysis, and optimization. In fact, any compact convex set is the convex hull of its extreme point set, and the result is called Krein-Milman theorem. The notion of a dentable subset of a Banach space was introduced by Rieffel [1] in conjunction with a Radon-Nikodym theorem for Banach space-valued measures. Subsequent work by Maynard [2] and by Davis and Phelps [3] has shown that those Banach spaces in which Rieffels Radon-Nikodym theorem is valid are precisely the ones in which every bounded closed convex set is dentable. This is a real breakthrough in studying the nature of Radon-Nikodym as a geometric property. In 1988, Bor-Luh Lin, Pei-Kee Lin, and S. L. Troyanski [4] described the characteristic of denting points and obtained the notion that there is a close relationship between denting points and strongly extreme points. It is easy to see that every denting point of $\mathrm{K}$ is a strongly extreme point of $\mathrm{K}$ and it is known [Ken Kunen and Haskeil Rosenthal, Martingale proofs of some geometric results in Banach space theory, Pacific J. Math. 100 (1982), 153-175] that every strongly extreme point of $\mathrm{K}$ is a weak*-extreme point of $\mathrm{K}$. Orlicz space is a special kind of Banach space; it was introduced by the famous Polish mathematician W. Orlicz in 1932. The theory of Orlicz space $[5,6]$ has been greatly developed because of its important theoretical properties and application value. Up to now, the criterion of an element in the unit sphere of Orlicz spaces equipped with the Orlicz norm $[5,7]$, the Luxemburg norm [5], and p-Amemiya norm [8] has been given. In this paper, we use a new technique to study the strongly extreme point in Orlicz spaces generated by Orlicz function and equipped with a new norm, namely, s-norm. The criterion of strongly extreme points is given, and the sufficient and necessary conditions for middle point locally uniformly convex in Orlicz spaces equipped with s-norm are obtained.

\section{Preliminaries}

Throughout this paper, $X$ will denote a Banach space and $X^{*}$ stands for the dual space of $X$. We denote by $(G, \Sigma, \mu)$ the nonatomic $\Sigma$-measure finite space. By $B(X)$ and $S(X)$ we denote the unit ball and the unit sphere of $X$, respectively. By $R$ and $N$ we will denote the sets of real and natural numbers, respectively.

A mapping $\Phi: R \longrightarrow[0, \infty)$ is said to be an Orlicz function if it is even, continuous, convex, and $\Phi(0)=0$, $\lim _{u \rightarrow \infty} \Phi(u)=\infty$. Moreover, if $\Phi$ satisfies $\lim _{u \rightarrow 0}(\Phi(u) /$ $u)=0$ and $\lim _{u \rightarrow \infty}(\Phi(u) / u)=\infty$, $\Phi$ is called $N$ function. Let $p_{+}(t)$ be the right-hand derivative of $\Phi$, where the function $\Psi$ is defined by the formula

$$
\Psi(u)=\sup \{|u| v-\Phi(v): v \geq 0\}
$$


and called complementary function to $\Phi$ in the sense of Young.

We say that an Orlicz function $\Phi$ satisfies $\triangle_{2}$-condition for large $u \in R\left(\Phi \in \triangle_{2}\right.$ for short) if there exist $u_{0}>0$ and $K>2$ such that

$$
\Phi(2 u) \leq K \Phi(u)
$$

whenever $|u|>u_{0}$.

Let $L^{0}$ denote the set of all measure real functions on $G$. For a given Orlicz function $\Phi$ we define on $L^{0}$ a convex function $I_{\Phi}: L^{0} \longrightarrow[0, \infty]$ (called a pseudomodular; see [6]) by

$$
I_{\Phi}(x)=\int_{G} \Phi(x(t)) d t
$$

It is clear that $I_{\Phi}(x)=\int_{\operatorname{supp}(x)} \Phi(x(t)) d t$; here $\operatorname{supp}(x)=\{t \in$ $G:|x(t)| \neq 0\}$.

The Orlicz space $L_{\Phi}$ generated by an Orlicz function $\Phi$ is defined by the formula

$$
L_{\Phi}=\left\{x \in L^{0}: I_{\Phi}(\lambda x)<+\infty \text { for some } \lambda>0\right\},
$$

and its subspace $E_{\Phi}$ is defined by

$$
E_{\Phi}=\left\{x \in L^{0}: I_{\Phi}(\lambda x)<+\infty \text { for all } \lambda>0\right\} .
$$
[5]

This space is usually equipped with the Luxemburg norm

$$
\|x\|=\inf \left\{k>0: I_{\Phi}\left(\frac{x}{k}\right) \leq 1\right\},
$$

or with the Orlicz norm (Amemiya norm) [5]

$$
\|x\|_{\Phi}^{\mathrm{o}}=\inf _{k>0} \frac{1}{k}\left(1+I_{\Phi}(k x)\right) .
$$

A function $s:[0, \infty) \longrightarrow[1, \infty)$ will be called an outer function, if it is convex and

$$
\max \{1, u\} \leq s(u) \leq 1+u \text { for all } u \geq 0 .
$$

In 2017, M.Wisła introduced $s$-norm.

Definition 1. Let $s$ be an outer function. Denote $s$-norm on Orlicz spaces by the formula

$$
\|x\|_{\Phi, s}=\inf _{k>0} \frac{1}{k} s\left(I_{\Phi}(k x)\right)
$$

It is easy to get $\|x\|_{\Phi, s}=\|x\|$ if $s(u)=\max \{1, u\}$ and $\|x\|_{\Phi, s}=\|x\|_{\Phi}^{o}$ if $s(u)=1+u([8])$. Then we have $\|x\| \leq$ $\|x\|_{\Phi, s} \leq\|x\|_{\Phi}^{o}$.

In this paper, by $L_{\Phi, s}$ we will denote an Orlicz space equipped with the $s$-norm.

Definition 2. Let $s_{+}^{\prime}(u)$ be the right-hand derivative of $s$. For all $0 \leq v \leq 1$, define

$$
\omega(v)=\int_{0}^{v} s_{+}^{\prime^{-1}}(t) d t
$$

Definition 3. Let $s$ be an outer function. For all $0 \leq u<\infty$ and $0 \leq v<\infty$,

$$
\beta_{s}(u, v)=1-\omega\left(s_{+}^{\prime}(u)\right)-v s_{+}^{\prime}(u),
$$

the function $\beta_{s}(u, v)$ is nonincreasing. For any $x \in L_{\Phi, s} \backslash\{0\}$, define ([9])

$$
\begin{aligned}
& k^{*}(x) \\
& =\inf \left\{k>0: \beta_{s}\left(I_{\Phi}(k x), I_{\Psi}\left(p_{+}(k|x|)\right)\right) \leq 0\right\}, \\
& k^{* *}(x) \\
& =\inf \left\{k>0: \beta_{s}\left(I_{\Phi}(k x), I_{\Psi}\left(p_{+}(k|x|)\right)\right) \geq 0\right\} . \\
& \text { Let } K(x)=\left[k^{*}(x), k^{* *}(x)\right] \text {. Then }\|x\|_{\Phi, s}=(1 / k) s\left(I_{\Phi}(k x)\right)
\end{aligned}
$$

Definition 4. A point $x \in S(X)$ is said to be an extreme point of $B(X)$ if for any $y, z \in B(X)$ with $x=(y+z) / 2$, then implies $y=z$.

The set of all extreme points of the unit ball $B(X)$ will be denoted by $\operatorname{Ext} B(X) . X$ is said to be strictly convex if $\operatorname{Ext} B(X)=S(X)$.

Definition 5. A point $x \in S(X)$ is said to be a strongly extreme point of $B(X)$ if for any $\left\{x_{n}\right\} \subseteq X,\left\{y_{n}\right\} \subseteq X$ with $\left\|x_{n}\right\| \longrightarrow 1$, $\left\|y_{n}\right\| \longrightarrow 1$ and $\left(x_{n}+y_{n}\right) / 2=x$ there holds $\left\|x_{n}-y_{n}\right\| \longrightarrow 0$ as $n \longrightarrow \infty$.

It is obvious that a strongly extreme point is an extreme point. $X$ is said a middle point locally uniformly convex Banach space if and only if each point on $S(X)$ is a strongly extreme point.

Definition 6. Let $u_{0}>0$. If for every $v, w \in R$ such that $v \neq w$ and $(v+w) / 2=u_{0}$, we have $\Phi\left(u_{0}\right)<(1 / 2) \Phi(v)+(1 / 2) \Phi(w)$, then $u_{0}$ is called to be a strictly convex point of $\Phi(u)$. The set of all strictly convex points of $\Phi(u)$ will be denoted by $S_{\Phi}$.

For the results concerning strongly extreme points and convexities in Orlicz spaces which are generated by $N$ function and equipped with the Orlicz norm, the Luxemburg norm, and p-Amemiya norm, we refer a reader to [10-17].

\section{Main Theorem}

Lemma 7. (1) If $\lim _{u \rightarrow \infty}(\Phi(u) / u)=\infty$ then $K(x) \neq \emptyset$ for any $x \in L_{\Phi, s} \backslash\{0\}$;

(2) If $\lim _{u \rightarrow \infty}(\Phi(u) / u)=A<\infty$ and $K(x)=\emptyset$ then $\|x\|_{\Phi, s}=A\|x\|_{1}$.

Proof. (1) Suppose $\lim _{u \rightarrow \infty}(\Phi(u) / u)=\infty$. We have $\lim _{k \rightarrow \infty} I_{\Psi}\left(p_{+}(k|x|)\right)=\infty$. Since for any $0 \leq v \leq 1, \omega(v) \epsilon$ $[0,1]$, then

$$
\begin{aligned}
& \lim _{k \rightarrow \infty} \beta_{s}\left(I_{\Phi}(k x), I_{\Psi}\left(p_{+}(k|x|)\right)\right)=\lim _{k \rightarrow \infty}(1 \\
& \left.\quad-\omega\left(s_{+}^{\prime}\left(I_{\Phi}(k x)\right)\right)-I_{\Psi}\left(p_{+}(k|x|)\right) s_{+}^{\prime}\left(I_{\Phi}(k x)\right)\right) \\
& \quad<0 .
\end{aligned}
$$

So $k^{*}(x)<\infty$, whence $K(x) \neq \emptyset$. 
(2) By $K(x)=\emptyset$, we have $k^{*}(x)=\infty$, and then

$$
\begin{aligned}
\|x\|_{\Phi, s} & =\inf _{k>0} \frac{1}{k} s\left(I_{\Phi}(k x)\right)=\lim _{k \longrightarrow \infty} \frac{1}{k} s\left(I_{\Phi}(k x)\right) \\
& \leq \lim _{k \rightarrow \infty} \frac{1}{k}\left(1+I_{\Phi}(k x)\right) \\
& =\lim _{k \rightarrow \infty}\left(\frac{1}{k}+\int_{\operatorname{supp}(x)} \frac{\Phi(k x(t))}{k|x(t)|}|x(t)| d t\right) \\
& =A\|x\|_{1},
\end{aligned}
$$

and

$$
\begin{aligned}
\|x\|_{\Phi, s} & =\lim _{k \longrightarrow \infty} \frac{1}{k} s\left(I_{\Phi}(k x)\right) \geq \lim _{k \longrightarrow \infty} \frac{1}{k} I_{\Phi}(k x) \\
& =\lim _{k \longrightarrow \infty} \int_{\operatorname{supp}(x)} \frac{\Phi(k x(t))}{k|x(t)|}|x(t)| d t=A\|x\|_{1} .
\end{aligned}
$$

Therefore $\|x\|_{\Phi, s}=A\|x\|_{1}$.

Corollary 8. $K(x)=\emptyset$ if and only if $\mu(\operatorname{supp}(x))<(1-$ $\omega(1)) / \Psi(A)$ for any $x \in L_{\Phi, s} \backslash\{0\}$.

Proof. Necessity. We know that $I_{\Phi}(k x) \longrightarrow \infty$ as $k \rightarrow \infty$. By $I_{\Phi}(k x) \leq s\left(I_{\Phi}(k x)\right) \leq 1+I_{\Phi}(k x)$, we can get $1 \leq$ $\lim _{k \longrightarrow \infty} s_{+}^{\prime}\left(I_{\Phi}(k x)\right) \leq 1$. That is, $\lim _{k \rightarrow \infty} s_{+}^{\prime}\left(I_{\Phi}(k x)\right)=1$. By $K(x)=\emptyset$, we have $k^{*}(x)=\infty$. Then

$$
\beta_{s}\left(I_{\Phi}(k x), I_{\Psi}\left(p_{+}(k|x|)\right)\right)>0,
$$

for all $k>0$. Since $\beta_{s}(u, v)$ is nonincreasing, we have

$$
\begin{aligned}
& \lim _{k \rightarrow \infty} \beta_{s}\left(I_{\Phi}(k x), I_{\Psi}\left(p_{+}(k|x|)\right)\right)=\lim _{k \longrightarrow \infty}(1 \\
& \left.-\omega\left(s_{+}^{\prime}\left(I_{\Phi}(k x)\right)\right)-I_{\Psi}\left(p_{+}(k|x|)\right) s_{+}^{\prime}\left(I_{\Phi}(k x)\right)\right) \\
& \quad=1-\omega(1)-\operatorname{supp}(x) \Psi(A)>0,
\end{aligned}
$$

whence $\mu(\operatorname{supp}(x))<(1-\omega(1)) / \Psi(A)$.

Here we infer that $\omega(1)<1$. If $\omega(1)=1$ we have $\lim _{k \rightarrow \infty} \beta_{s}\left(I_{\Phi}(k x), I_{\Psi}\left(p_{+}(k|x|)\right)\right)=-\operatorname{supp}(x) \Psi(A)<0$, a contradiction.

Sufficiency. By the definitions of $s(u)$ and $\omega(v), s_{+}^{\prime}(u) \leq 1$ and $\omega\left(s_{+}^{\prime}(u)\right) \leq \omega(1)$ for any $u>0$. Therefore for all $k>0$

$$
\begin{aligned}
\beta_{s}\left(I_{\Phi}(k x), I_{\Psi}\left(p_{+}(k|x|)\right)\right) \\
=1-\omega\left(s_{+}^{\prime}\left(I_{\Phi}(k x)\right)\right) \\
\quad-I_{\Psi}\left(p_{+}(k|x|)\right) s_{+}^{\prime}\left(I_{\Phi}(k x)\right) \\
\geq 1-\omega(1)-I_{\Psi}\left(p_{+}(k|x|)\right) \\
=1-\omega(1)-\mu(\operatorname{supp}(x)) \Psi(A)>0,
\end{aligned}
$$

whence $k^{*}(x)=\infty$, i.e., $K(x)=\emptyset$.

Theorem 9. Suppose that $s(u)>1$ when $u>0$ and $\Phi$ is an Orlicz function. A point $x_{0} \in S\left(L_{\Phi, s}\right)$ is a strongly extreme point if and only if $\Phi \in \triangle_{2}$ and $k_{0} x_{0}(t) \in S_{\Phi}$ for $k_{0} \in K\left(x_{0}\right)$.
Proof. Necessity. As we know that a strongly extreme point is an extreme point, we only need to prove that $x_{0} \in \operatorname{Ext} B\left(L_{\Phi, s}\right)$ implies $k_{0} x_{0}(t) \in S_{\Phi}$ for $k_{0} \in K\left(x_{0}\right)$. Firstly, we will prove that if $x_{0} \in \operatorname{ExtB}\left(L_{\Phi, s}\right)$, then $K\left(x_{0}\right) \neq \emptyset$. If $K\left(x_{0}\right)=\emptyset$, we will have $k^{*}\left(x_{0}\right)=\infty$ which implies that $\mu\left(\operatorname{supp}\left(x_{0}\right)\right)<(1-$ $\omega(1)) / \Psi(A)$ holds. There exists $a>0$ such that $\mu(\{t \in G$ : $\left.\left.\left|x_{0}(t)\right|>a\right\}\right)>0$. Put $C=\left\{t \in G:\left|x_{0}(t)\right|>a\right\}$ and $0<$ $\mu(C)<(1-\omega(1)) / \Psi(A)$. Divide $C$ into two sets $C_{1}$ and $C_{2}$ with $C_{1} \cap C_{2}=\emptyset$ and $\mu\left(C_{1}\right)=\mu\left(C_{2}\right)$. Take $\varepsilon \in(0, a)$ and define

$$
\begin{aligned}
& y(t)= \begin{cases}x_{0}(t), & t \in G \backslash\left(C_{1} \cup C_{2}\right) \\
x_{0}(t)-\varepsilon, & t \in C_{1} \\
x_{0}(t)+\varepsilon, & t \in C_{2},\end{cases} \\
& z(t)= \begin{cases}x_{0}(t), & t \in G \backslash\left(C_{1} \cup C_{2}\right) \\
x_{0}(t)+\varepsilon, & t \in C_{1} \\
x_{0}(t)-\varepsilon, & t \in C_{2} .\end{cases}
\end{aligned}
$$

Then $x_{0}=(y+z) / 2, y \neq z$. Moreover $\operatorname{supp}(y) \subseteq \operatorname{supp}\left(x_{0}\right)$, $\operatorname{supp}(z) \subseteq \operatorname{supp}\left(x_{0}\right)$. We have

$$
\begin{aligned}
& \|y\|_{\Phi, s}=A\|y\|_{1}=A \int_{G}|y(t)| d t \\
& =A\left(\int_{C_{1}}\left|x_{0}(t)-\varepsilon\right| d t+\int_{C_{2}}\left|x_{0}(t)+\varepsilon\right| d t\right. \\
& \left.\quad+\int_{G \backslash\left(C_{1} \cup C_{2}\right)}\left|x_{0}(t)\right| d t\right)=A \int_{G}\left|x_{0}(t)\right| d t \\
& =A\left\|x_{0}\right\|_{1}=\left\|x_{0}\right\|_{\Phi, s}=1 .
\end{aligned}
$$

Similarly, we can get $\|z\|_{\Phi, s}=1$.

Next we will show that $k_{0} x_{0}(t) \in S_{\Phi}$.

Suppose that $\mu\left(\left\{t \in G: k_{0} x_{0}(t) \notin S_{\Phi}\right\}\right)>0$ for $k_{0} \epsilon$ $K\left(x_{0}\right)$. There exists an interval $(a, b)$ such that $\mu(\{t \in G$ : $\left.\left.a / k_{0}+\varepsilon<x_{0}(t)<b / k_{0}-\varepsilon\right\}\right)>0(\varepsilon>0)$, and $\Phi$ is affine on $(a, b): \Phi(x)=p x+q$. Divide $\left\{t \in G: a / k_{0}+\varepsilon<x_{0}(t)<\right.$ $\left.b / k_{0}-\varepsilon\right\}$ into two sets $E$ and $F$ with $E \cap F=\emptyset$ and $\mu(E)=\mu(F)$. Define

$$
\begin{aligned}
& y(t)= \begin{cases}x_{0}(t), & t \in G \backslash(E \cup F) \\
x_{0}(t)-\varepsilon, & t \in E \\
x_{0}(t)+\varepsilon, & t \in F,\end{cases} \\
& z(t)= \begin{cases}x_{0}(t), & t \in G \backslash(E \cup F) \\
x_{0}(t)+\varepsilon, & t \in E \\
x_{0}(t)-\varepsilon, & t \in F .\end{cases}
\end{aligned}
$$

Then $x_{0}=(y+z) / 2, y \neq z$. Thus

$$
\begin{aligned}
I_{\Phi}\left(k_{0} y\right)= & \int_{E \cup F} \Phi\left(k_{0} y(t)\right) d t \\
& +\int_{G \backslash(E \cup F)} \Phi\left(k_{0} y(t)\right) d t
\end{aligned}
$$




$$
\begin{aligned}
= & \int_{E}\left(p\left(k_{0}\left(x_{0}(t)-\varepsilon\right)\right)+q\right) d t \\
& +\int_{F}\left(p\left(k_{0}\left(x_{0}(t)+\varepsilon\right)\right)+q\right) d t \\
& +\int_{G \backslash(E \cup F)} \Phi\left(k_{0} x_{0}(t)\right) d t \\
= & \int_{E \cup F}\left(p k_{0} x_{0}(t)+q\right) d t \\
& +\int_{G \backslash(E \cup F)} \Phi\left(k_{0} x_{0}(t)\right) d t \\
= & \int_{E \cup F} \Phi\left(k_{0} x_{0}(t)\right) d t \\
& +\int_{G \backslash(E \cup F)} \Phi\left(k_{0} x_{0}(t)\right) d t=I_{\Phi}\left(k_{0} x_{0}\right),
\end{aligned}
$$

whence $\|y\|_{\Phi, s} \leq\left(1 / k_{0}\right) s\left(I_{\Phi}\left(k_{0} y\right)\right)=\left(1 / k_{0}\right) s\left(I_{\Phi}\left(k_{0} x_{0}\right)\right)=$ $\left\|x_{0}\right\|_{\Phi, s}=1$. In the same way, we can prove $\|z\|_{\Phi, s} \leq 1$. This contradicts the fact that $x_{0}$ is an extreme point of $B\left(L_{\Phi, s}\right)$.

In order to complete this proof, we need to prove that if $\Phi \notin \triangle_{2}$ there are no strongly extreme points in $S\left(L_{\Phi, s}\right)$.

Suppose $\Phi \notin \triangle_{2}$. Then $\lim _{u \rightarrow \infty}(\Phi(u) / u)=+\infty$.

In fact, if $\lim _{u \rightarrow \infty}(\Phi(u) / u)=A<+\infty$, there exists $u_{0}>$ 0 such that $(A / 2) u<\Phi(u)<(3 A / 2) u$ holds for every $u>u_{0}$. Then we have $\Phi(2 u)<(3 / 2) A(2 u)<6(A / 2) u \leq 6 \Phi(u)$; it implies $\Phi \in \triangle_{2}$, a contradiction.

For any $x_{0} \in S\left(L_{\Phi, s}\right)$, there exists $k_{0}>0$ such that

$$
1=\left\|x_{0}\right\|_{\Phi, s}=\frac{1}{k_{0}} s\left(I_{\Phi}\left(k_{0} x_{0}\right)\right) .
$$

Since $x_{0} \in S\left(L_{\Phi, s}\right)$, we can find $d>0$ such that $\mu(\{t \in G$ : $\left.\left.\left|x_{0}(t)\right| \leq d\right\}\right)>0$. By $\Phi \notin \triangle_{2}$, there exist $u_{n}>0$ and $u_{n} \uparrow \infty$ such that $\Phi\left(2 u_{n}\right)>2^{n} \Phi\left(u_{n}\right)(n=1,2, \cdots)$. We may assume that $1 / \Phi\left(u_{1}\right)<\mu\left(\left\{t \in G:\left|x_{0}(t)\right| \leq d\right\}\right)$. Take $\left\{G_{n}\right\} \subset\{t \in$ $\left.G:\left|x_{0}(t)\right| \leq d\right\}$ with $G_{m} \cap G_{n}=\emptyset$ for any $m \neq n$, satisfying $\mu\left(G_{n}\right)=1 /\left(2^{n} \Phi\left(u_{n}\right)\right)(n=1,2, \cdots)$. Define

$$
\begin{aligned}
& x_{n}(t)= \begin{cases}x_{0}(t), & t \in G \backslash G_{n} \\
x_{0}(t)+\frac{u_{n}}{k_{0}}, & t \in G_{n},\end{cases} \\
& y_{n}(t)= \begin{cases}x_{0}(t), & t \in G \backslash G_{n} \\
x_{0}(t)-\frac{u_{n}}{k_{0}}, & t \in G_{n} .\end{cases}
\end{aligned}
$$

Then $x_{0}=\left(x_{n}+y_{n}\right) / 2, x_{n}(t)=x_{n}^{\prime}(t)+x_{n}^{\prime \prime}(t)$, here $x_{n}^{\prime}(t)=$ $x_{0} \chi_{G \backslash G_{n}}(t)+\left(u_{n} / k_{0}\right) \chi_{G_{n}}(t), x_{n}^{\prime \prime}(t)=x_{0} \chi_{G_{n}}(t)$.

Notice that

$$
\left\|x_{n}^{\prime \prime}\right\|_{\Phi, s}=\left\|x_{0} \chi_{G_{n}}\right\|_{\Phi, s} \leq d\left\|\chi_{G_{n}}\right\|_{\Phi, s} \longrightarrow 0
$$

We have $\left\|x_{n}^{\prime}\right\|_{\Phi, s} \geq\left\|x_{0} \chi_{G \backslash G_{n}}\right\|_{\Phi, s} \geq\left\|x_{0}\right\|_{\Phi, s}-\left\|x_{0} \chi_{G_{n}}\right\|_{\Phi, s}$, that is, $\underline{\lim }_{n \rightarrow \infty}\left\|x_{n}^{\prime}\right\|_{\Phi, s} \geq\left\|x_{0}\right\|_{\Phi, s}=1$. And

$$
\begin{aligned}
& \left\|x_{n}^{\prime}\right\|_{\Phi, s}=\inf _{k>0} \frac{1}{k} s\left(I_{\Phi}\left(k x_{n}^{\prime}\right)\right) \leq \frac{1}{k_{0}} s\left(I_{\Phi}\left(k_{0} x_{n}^{\prime}\right)\right)=\frac{1}{k_{0}} \\
& \quad \cdot s\left(\int_{G} \Phi\left(k_{0}\left(x_{0} \chi_{G \backslash G_{n}}(t)+\frac{u_{n}}{k_{0}} \chi_{G_{n}}(t)\right)\right) d t\right) \\
& \quad=\frac{1}{k_{0}} s\left(\int_{G \backslash G_{n}} \Phi\left(k_{0} x_{0} \chi_{G \backslash G_{n}}(t)\right) d t\right. \\
& \left.\quad+\int_{G_{n}} \Phi\left(u_{n} \chi_{G_{n}}(t)\right) d t\right) \leq \frac{1}{k_{0}} s\left(I_{\Phi}\left(k_{0} x_{0}\right)\right. \\
& \left.\quad+\Phi\left(u_{n}\right) \mu\left(G_{n}\right)\right)=\frac{1}{k_{0}} s\left(I_{\Phi}\left(k_{0} x_{0}\right)+\frac{1}{2^{n}}\right) .
\end{aligned}
$$

Consequently, $\overline{\lim }_{n \longrightarrow \infty}\left\|x_{n}^{\prime}\right\|_{\Phi, s} \leq\left\|x_{0}\right\|_{\Phi, s}=1$. Hence $\lim _{n \rightarrow \infty}\left\|x_{n}\right\|_{\Phi, s}=1$. In the same way, we have $\lim _{n \longrightarrow \infty}\left\|y_{n}\right\|_{\Phi, s}=1$. But $I_{\Phi}\left(k_{0}\left(x_{n}-y_{n}\right)\right)=\int_{G_{n}} \Phi\left(k_{0}\left(2 u_{n}(t) /\right.\right.$ $\left.\left.k_{0}\right)\right) d t=\Phi\left(2 u_{n}\right) \mu\left(G_{n}\right) \geq 1(n=1,2, \cdots)$, which implies $\left\|x_{n}-y_{n}\right\|_{\Phi, s}=\left(1 / k_{0}\right)\left\|2 u_{n} \chi_{G_{n}}\right\|_{\Phi, s} \geq\left(1 / k_{0}\right)\left\|2 u_{n} \chi_{G_{n}}\right\| \geq 1 / k_{0}$, a contradiction.

Sufficiency. Let $\Phi \in \triangle_{2}, x_{0} \in S\left(L_{\Phi, s}\right)$ with $k_{0} x_{0}(t) \in S_{\Phi}$ for $k_{0} \in K\left(x_{0}\right)$. Take any $x_{n}, y_{n} \in L_{\Phi, s}$ with $\left\|x_{n}\right\|_{\Phi, s} \longrightarrow$ $1,\left\|y_{n}\right\|_{\Phi, s} \longrightarrow 1$ and $x_{n}+y_{n}=2 x_{0}$.

Take sequences $\left\{k_{n}\right\}$ and $\left\{h_{n}\right\}$ of positive numbers such that

$$
\begin{aligned}
\left\|x_{n}\right\|_{\Phi, s} & \geq \frac{1}{k_{n}} s\left(I_{\Phi}\left(k_{n} x_{n}\right)\right)-\frac{1}{n}, \\
\left\|y_{n}\right\|_{\Phi, s} & \geq \frac{1}{h_{n}} s\left(I_{\Phi}\left(h_{n} y_{n}\right)\right)-\frac{1}{n} .
\end{aligned}
$$

Define

$$
\begin{aligned}
x_{n}^{\prime} & =\frac{x_{n}+x_{0}}{2} \\
\text { and } y_{n}^{\prime} & =\frac{y_{n}+x_{0}}{2},
\end{aligned}
$$

then $x_{n}^{\prime}+y_{n}^{\prime}=2 x_{0}$ and $\overline{\lim }_{n \longrightarrow \infty}\left\|x_{n}^{\prime}\right\|_{\Phi, s} \leq 1, \varlimsup_{n \rightarrow \infty}\left\|y_{n}^{\prime}\right\|_{\Phi, s}$ $\leq 1$.

Now we will prove that $\lim _{n \longrightarrow \infty}\left\|x_{n}^{\prime}\right\|_{\Phi, s}=1$ and $\lim _{n \rightarrow \infty}\left\|y_{n}^{\prime}\right\|_{\Phi, s}=1$. Otherwise, we can assume that $\lim _{n \rightarrow \infty}\left\|x_{n}^{\prime}\right\|_{\Phi, s}<1$; there exist $\delta>0$ and $n_{0} \in N$ such that, for every $n \geq n_{0}$,

$$
\begin{aligned}
\left\|x_{n}^{\prime}\right\|_{\Phi, s} & \leq 1-\delta, \\
\left\|y_{n}^{\prime}\right\|_{\Phi, s} & \leq 1+\frac{\delta}{2} .
\end{aligned}
$$

Then

$$
\begin{aligned}
1 & =\left\|x_{0}\right\|_{\Phi, \mathrm{s}}=\left\|\frac{x_{n}^{\prime}+y_{n}^{\prime}}{2}\right\|_{\Phi, s} \leq \frac{1}{2}\left(1-\delta+1+\frac{\delta}{2}\right) \\
& <1
\end{aligned}
$$

a contradiction. 
Since $\left\|x_{n}^{\prime}-y_{n}^{\prime}\right\|_{\Phi, s} \longrightarrow 0$ if and only if $\left\|x_{n}-y_{n}\right\|_{\Phi, s} \longrightarrow$ $0(n \longrightarrow \infty)$, we will consider the sequences $\left\{x_{n}^{\prime}\right\}$ and $\left\{y_{n}^{\prime}\right\}$, where $\left\{x_{n}^{\prime}\right\}$ and $\left\{y_{n}^{\prime}\right\}$ in place of $\left\{x_{n}\right\}$ and $\left\{y_{n}\right\}$.

Put $k_{n}^{\prime}=2 k_{n} k_{0} /\left(k_{n}+k_{0}\right)$ and $h_{n}^{\prime}=2 h_{n} k_{0} /\left(h_{n}+k_{0}\right)$. Then $\left\{k_{n}^{\prime}\right\}$ and $\left\{h_{n}^{\prime}\right\}$ are bounded. Since $\left\|x_{n}^{\prime}\right\|_{\Phi, s} \longrightarrow 1(n \longrightarrow \infty)$, we have

$$
\begin{aligned}
1 & \longleftarrow\left\|x_{n}^{\prime}\right\|_{\Phi, s} \leq \frac{1}{k_{n}^{\prime}} s\left(I_{\Phi}\left(k_{n}^{\prime} x_{n}^{\prime}\right)\right. \\
& =\frac{k_{n}+k_{0}}{2 k_{n} k_{0}} s\left(I_{\Phi}\left(\frac{k_{n} k_{0}}{k_{n}+k_{0}}\left(x_{n}+x_{0}\right)\right)\right. \\
& \leq \frac{1}{2}\left(\frac{1}{k_{0}} s\left(I_{\Phi}\left(k_{0} x_{0}\right)\right)+\frac{1}{k_{n}} s\left(I_{\Phi}\left(k_{n} x_{n}\right)\right)\right) \\
& \leq \frac{1}{2}\left(\left\|x_{0}\right\|_{\Phi, s}+\left\|x_{n}\right\|_{\Phi, s}+\frac{1}{n}\right) \longrightarrow 1 \quad(n \longrightarrow \infty),
\end{aligned}
$$

whence it follows that

$$
\frac{1}{k_{n}^{\prime}} s\left(I_{\Phi}\left(k_{n}^{\prime} x_{n}^{\prime}\right)\right) \longrightarrow 1 \quad(n \longrightarrow \infty) .
$$

Analogously,

$$
\frac{1}{h_{n}^{\prime}} s\left(I_{\Phi}\left(h_{n}^{\prime} y_{n}^{\prime}\right)\right) \longrightarrow 1 \quad(n \longrightarrow \infty) .
$$

Put $d=\sup _{n}\left\{k_{n}^{\prime}, h_{n}^{\prime}\right\}<\infty$. Assume that $k_{n}^{\prime} \longrightarrow k$ and $h_{n}^{\prime} \longrightarrow h$ as $n \longrightarrow \infty$. Now we prove $k, h \geq 1$. Since

$$
1 \longleftarrow \frac{1}{k_{n}^{\prime}} s\left(I_{\Phi}\left(k_{n}^{\prime} x_{n}^{\prime}\right)\right) \quad(n \longrightarrow \infty),
$$

then

$$
s\left(I_{\Phi}\left(k_{n}^{\prime} x_{n}^{\prime}\right)\right) \longrightarrow k \quad(n \longrightarrow \infty),
$$

and if $k<1$, consequently, $s\left(I_{\Phi}\left(k_{n}^{\prime} x_{n}^{\prime}\right)\right)<1$ as $n \longrightarrow \infty$, a contradiction. Thus $k \geq 1$. Similarly, $h \geq 1$. Then we have $k /(k+h), h /(k+h) \in[1 /(1+d), d /(1+d)]$.

Step 1. We will show that $k_{0}=2 k h /(k+h)$. In fact

$$
\begin{aligned}
& 1=\left\|x_{0}\right\|_{\Phi, s}=\frac{1}{k_{0}} s\left(I_{\Phi}\left(k_{0} x_{0}\right)\right) \leq \frac{k_{n}^{\prime}+h_{n}^{\prime}}{2 k_{n}^{\prime} h_{n}^{\prime}} \\
& \cdot s\left(I_{\Phi}\left(\frac{2 k_{n}^{\prime} h_{n}^{\prime}}{k_{n}^{\prime}+h_{n}^{\prime}} x_{0}\right)\right) \leq \frac{k_{n}^{\prime}+h_{n}^{\prime}}{2 k_{n}^{\prime} h_{n}^{\prime}} \\
& \cdot s\left(I_{\Phi}\left(\frac{k_{n}^{\prime} h_{n}^{\prime}}{k_{n}^{\prime}+h_{n}^{\prime}}\left(x_{n}^{\prime}+y_{n}^{\prime}\right)\right)\right) \leq \frac{k_{n}^{\prime}+h_{n}^{\prime}}{2 k_{n}^{\prime} h_{n}^{\prime}} \\
& \cdot s\left(I_{\Phi}\left(\frac{h_{n}^{\prime}}{k_{n}^{\prime}+h_{n}^{\prime}} k_{n}^{\prime} x_{n}^{\prime}\right)+I_{\Phi}\left(\frac{k_{n}^{\prime}}{k_{n}^{\prime}+h_{n}^{\prime}} h_{n}^{\prime} y_{n}^{\prime}\right)\right) \\
& \leq \frac{1}{2}\left(\frac{1}{k_{n}^{\prime}} s\left(I_{\Phi}\left(k_{n}^{\prime} x_{n}^{\prime}\right)\right)+\frac{1}{h_{n}^{\prime}} s\left(I_{\Phi}\left(h_{n}^{\prime} y_{n}^{\prime}\right)\right)\right) \longrightarrow 1 \\
& \quad(n \longrightarrow \infty),
\end{aligned}
$$

whence $2 k_{n}^{\prime} h_{n}^{\prime} /\left(k_{n}^{\prime}+h_{n}^{\prime}\right) \longrightarrow 2 k h /(k+h)=k_{0} \in K\left(x_{0}\right)$ as $n \longrightarrow \infty$.

Step 2. We will show that $k_{n}^{\prime} x_{n}^{\prime}-k_{0} x_{0} \stackrel{\mu}{\rightarrow} 0(n \longrightarrow \infty)$.

Firstly, we will prove that $k x_{n}^{\prime}-h y_{n}^{\prime} \stackrel{\mu}{\rightarrow} 0(n \longrightarrow \infty)$. Otherwise, there exist $\sigma_{0}, \varepsilon_{0}>0$ such that $\mu\left(\left\{t \in G: \mid k x_{n}^{\prime}(t)-\right.\right.$ $\left.\left.h y_{n}^{\prime}(t) \mid \geq \sigma_{0}\right\}\right) \geq \varepsilon_{0}$. Let $D=\Phi^{-1}\left(3 / \varepsilon_{0}\right)$ and $D_{1}=2 k D$. Let $G_{n}=\left\{t \in G:\left|k x_{n}^{\prime}(t)\right| \leq D_{1},\left|h y_{n}^{\prime}(t)\right| \leq D_{1},\left|k x_{n}^{\prime}(t)-h y_{n}^{\prime}(t)\right| \geq\right.$ $\left.\sigma_{0}\right\}$. It can be easy to calculate that $\mu\left(G_{n}\right)>\varepsilon_{0} / 3$. In fact, since $\lim _{n \rightarrow \infty}\left\|x_{n}^{\prime}\right\|_{\Phi, s}=1,\left\{x_{n}^{\prime}\right\}$ is bounded in norm. Without loss of generality, we may assume that $2 \geq\left\|x_{n}^{\prime}\right\|_{\Phi, s} \geq\left\|x_{n}^{\prime}\right\|$; then

$$
\begin{aligned}
1 & \geq 1_{\Phi}\left(\frac{x_{n}^{\prime}}{2}\right)>\int_{\left\{t \in G:\left|x_{n}^{\prime}(t) / 2\right|>D\right\}} \Phi\left(\frac{x_{n}^{\prime}(t)}{2}\right) d t \\
& >\Phi(D) \mu\left(\left\{t \in G:\left|\frac{x_{n}^{\prime}(t)}{2}\right|>D\right\}\right) \\
& =\frac{3}{\varepsilon_{0}} \mu\left(\left\{t \in G:\left|\frac{x_{n}^{\prime}(t)}{2}\right|>D\right\}\right),
\end{aligned}
$$

whence $\mu\left(\left\{t \in G:\left|x_{n}^{\prime}(t) / 2\right|>D\right\}\right)<\varepsilon_{0} / 3$. We have $\mu(\{t \in G$ : $\left.\left.\left|k x_{n}^{\prime}(t)\right|>D_{1}\right\}\right)<\varepsilon_{0} / 3$. Hence

$$
\begin{aligned}
\mu\left(G_{n}\right) \geq & \mu\left(\left\{t \in G:\left|k x_{n}^{\prime}(t)-h y_{n}^{\prime}(t)\right| \geq \sigma_{0}\right\}\right) \\
& -\mu\left(\left\{t \in G:\left|k x_{n}^{\prime}(t)\right|>D_{1}\right\}\right) \\
& -\mu\left(\left\{t \in G:\left|h y_{n}^{\prime}(t)\right|>D_{1}\right\}\right) \\
> & \varepsilon_{0}-\frac{\varepsilon_{0}}{3}-\frac{\varepsilon_{0}}{3}=\frac{\varepsilon_{0}}{3} .
\end{aligned}
$$

We know that $S_{\Phi}$ is a close set. Let

$$
\begin{aligned}
F & =\left\{(x, y):|x| \leq D_{1},|y| \leq D_{1}, \mid x-y\right) \mid \\
& \left.\geq \sigma_{0}, \frac{h}{k+h} x+\frac{k}{k+h} y \in S_{\Phi}\right\} .
\end{aligned}
$$

$F$ is a bounded close set. For every $(x, y) \in F$, the continuous function is

$$
\frac{\Phi((h /(k+h)) x+(k /(k+h)) y)}{(h /(k+h)) \Phi(x)+(k /(k+h)) \Phi(y)}<1 .
$$

Set maximum value equal to $1-\delta(\delta>0)$. For every $(x, y) \in F$, we have

$$
\begin{aligned}
& \Phi\left(\frac{h}{k+h} x+\frac{k}{k+h} y\right) \\
& \quad \leq(1-\delta)\left(\frac{h}{k+h} \Phi(x)+\frac{k}{k+h} \Phi(y)\right) .
\end{aligned}
$$

Since $k_{0} x_{0}(t) \in S_{\Phi}$, we have

$$
\begin{aligned}
& \frac{h}{k+h} k x_{n}^{\prime}(t)+\frac{k}{k+h} h y_{n}^{\prime}(t)=\frac{2 k h}{k+h} x_{0}(t)=k_{0} x_{0} \\
& \quad \in S_{\Phi}
\end{aligned}
$$


for $t \in G$. Therefore, $\left(k x_{n}^{\prime}(t), h y_{n}^{\prime}(t)\right) \in F$, i.e., for $t \in G_{n}$, and

$$
\begin{aligned}
& \Phi\left(\frac{h}{k+h} k x_{n}^{\prime}(t)+\frac{k}{k+h} h y_{n}^{\prime}(t)\right) \\
& \quad \leq(1-\delta)\left(\frac{h}{k+h} \Phi\left(k x_{n}^{\prime}(t)\right)+\frac{k}{k+h} \Phi\left(h y_{n}^{\prime}(t)\right)\right) .
\end{aligned}
$$

Hence

$$
\begin{aligned}
& \left\|x_{n}^{\prime}+y_{n}^{\prime}\right\|_{\Phi, s} \leq \frac{k+h}{k h} s\left(I_{\Phi}\left(\frac{k h}{k+h}\left(x_{n}^{\prime}+y_{n}^{\prime}\right)\right)\right) \\
& \quad \leq \frac{k+h}{k h} s\left(\int_{G} \Phi\left(\frac{k h}{k+h}\left(x_{n}^{\prime}(t)+y_{n}^{\prime}(t)\right)\right) d t\right) \\
& \quad \leq \frac{k+h}{k h} s\left(( 1 - \delta ) \int _ { G _ { n } } \left[\frac{h}{k+h} \Phi\left(k x_{n}^{\prime}(t)\right)+\frac{k}{k+h}\right.\right. \\
& \left.\cdot \Phi\left(h y_{n}^{\prime}(t)\right)\right] d t+\int_{G \backslash G_{n}}\left[\frac{h}{k+h} \Phi\left(k x_{n}^{\prime}(t)\right)\right. \\
& \left.\left.+\frac{k}{k+h} \Phi\left(h y_{n}^{\prime}(t)\right)\right] d t\right)=\frac{k+h}{k h} \\
& \cdot s\left(\int_{G}\left[\frac{h}{k+h} \Phi\left(k x_{n}^{\prime}(t)\right)+\frac{k}{k+h} \Phi\left(h y_{n}^{\prime}(t)\right)\right] d t\right. \\
& -\delta \int_{G_{n}}\left[\frac{h}{k+h} \Phi\left(k x_{n}^{\prime}(t)\right)+\frac{k}{k+h}\right. \\
& \left.\left.\cdot \Phi\left(h y_{n}^{\prime}(t)\right)\right] d t\right) \leq \frac{1}{k} s\left(I_{\Phi}\left(k x_{n}^{\prime}\right)\right)+\frac{1}{h} \\
& \cdot s\left(I_{\Phi}\left(h y_{n}^{\prime}\right)\right)-\frac{k+h}{k h}\left(s \left(\delta \int _ { G _ { n } } \left[\frac{h}{k+h}\right.\right.\right. \\
& \left.\left.\left.\cdot \Phi\left(k x_{n}^{\prime}(t)\right)+\frac{k}{k+h} \Phi\left(h y_{n}^{\prime}(t)\right)\right] d t\right)-1\right) .
\end{aligned}
$$

Notice that

$$
\begin{aligned}
& I_{\Phi}\left(\left(k-k_{n}^{\prime}\right) x_{n}^{\prime}\right) \leq\left|k-k_{n}^{\prime}\right| I_{\Phi}\left(x_{n}^{\prime}\right) \longrightarrow 0 \\
& (n \longrightarrow \infty) \text {. }
\end{aligned}
$$

Since $\Phi \in \triangle_{2}$, there holds

$$
\begin{aligned}
& I_{\Phi}\left(k x_{n}^{\prime}\right)-I_{\Phi}\left(k_{n}^{\prime} x_{n}^{\prime}\right) \\
& \quad=I_{\Phi}\left(k_{n}^{\prime} x_{n}^{\prime}+\left(k-k_{n}^{\prime}\right) x_{n}^{\prime}\right)-I_{\Phi}\left(k_{n}^{\prime} x_{n}^{\prime}\right) \longrightarrow 0 \\
& \quad(n \longrightarrow \infty) .
\end{aligned}
$$

Thus

$$
\begin{aligned}
& 0 \leq \frac{1}{k} s\left(I_{\Phi}\left(k x_{n}^{\prime}\right)\right)-\left\|x_{n}^{\prime}\right\|_{\Phi, s} \\
& \leq \frac{1}{k} s\left(I_{\Phi}\left(k x_{n}^{\prime}\right)\right)-\frac{1}{k_{n}^{\prime}} s\left(I_{\Phi}\left(k_{n}^{\prime} x_{n}^{\prime}\right)\right)+\frac{1}{n} \longrightarrow 0 \\
& \quad(n \longrightarrow \infty) .
\end{aligned}
$$

Similarly, we can get $(1 / h) s\left(I_{\Phi}\left(h y_{n}^{\prime}\right)\right)-\left\|y_{n}^{\prime}\right\|_{\Phi, s} \longrightarrow 0(n \longrightarrow$ $\infty)$. Then $\left\|x_{n}^{\prime}+y_{n}^{\prime}\right\|_{\Phi, s} \leq 2-((k+h) / k h)(s((2 \delta /(1+$ d)) $\left.\left.\Phi\left(\sigma_{0} / 2\right)\left(\varepsilon_{0} / 3\right)\right)-1\right)$ as $n \longrightarrow \infty$. By $s(u)>1$ when $u>0$, we have $\lim _{n \rightarrow \infty}\left\|x_{n}^{\prime}+y_{n}^{\prime}\right\|_{\Phi, s}<2$. The contradiction shows that $k x_{n}^{\prime}-h y_{n}^{\prime} \stackrel{\mu}{\rightarrow} 0$.

Since s-norm is equivalent with the Luxemburg norm, their weak topology and weak star topology are all equivalent. Then $L_{\Phi, s}$ is $w^{*}$ compact. Take $\left\{x_{n}^{\prime \prime}\right\} \subset\left\{x_{n}^{\prime}\right\},\left\{y_{n}^{\prime \prime}\right\} \subset\left\{y_{n}^{\prime}\right\}$ such that $x_{n}^{\prime \prime} \stackrel{w^{*}}{\longrightarrow} x^{\prime}$ and $y_{n}^{\prime \prime} \stackrel{w^{*}}{\longrightarrow} y^{\prime}$. We can get $x^{\prime}+y^{\prime}=2 x_{0}$. By

$$
\|x\|_{\Phi, s}=\sup \left\{\int_{G} x(t) y(t) d t: y \in B\left(L_{\Phi, s}^{*}\right)\right\},
$$

where $B\left(L_{\Phi, s}^{*}\right)$ represents the unit ball of the dual space of $E_{\Phi, s}$, we have $y \in L_{\Psi}$ and

$$
\|x\|_{\Phi, s} \geq \sup \left\{\int_{G} x(t) y(t) d t: y \in B\left(E_{\Phi, s}^{*}\right)\right\} .
$$

For any $\varepsilon>0$, there exists $y \in B\left(L_{\Phi, s}^{*}\right)$ such that

$$
\|x\|_{\Phi, s}-\varepsilon \leq \int_{G} x(t) y(t) d t .
$$

Put

$$
y_{n}(t)= \begin{cases}y(t), & |y(t)| \leq n \\ 0, & |y(t)|>n .\end{cases}
$$

Then $y_{n}(t) \in B\left(E_{\Phi, s}^{*}\right)$ and

$$
\int_{G} x(t) y(t) d t=\lim _{n \longrightarrow \infty} \int_{G} x(t) y_{n}(t) d t .
$$

By the definition of "lim", for any $\varepsilon>0$, there exists $n_{0} \in N$ such that

$$
\int_{G} x(t) y(t) d t-\varepsilon \leq \int_{G} x(t) y_{n}(t) d t
$$

whenever $n \geq n_{0}$.

Thus

$$
\|x\|_{\Phi, s}-2 \varepsilon \leq \int_{G} x(t) y_{n}(t) d t .
$$

By arbitrariness of $\varepsilon$ and combining with the above proof, we can obtain

$$
\|x\|_{\Phi, s}=\sup \left\{\int_{G} x(t) y(t) d t: y \in B\left(E_{\Phi, s}^{*}\right)\right\} .
$$

Therefore

$$
\begin{aligned}
2 & =\left\|2 x_{0}\right\|_{\Phi, s} \leq\left\|x^{\prime}\right\|_{\Phi, s}+\left\|y^{\prime}\right\|_{\Phi, s} \\
& \leq \varliminf_{n \Longrightarrow \infty}^{\lim }\left\|x_{n}^{\prime}\right\|_{\Phi, s}+\underline{\lim }_{n \Longrightarrow \infty}\left\|y_{n}^{\prime}\right\|_{\Phi, s}=2 .
\end{aligned}
$$

This shows $\left\|x^{\prime}\right\|_{\Phi, s}=\left\|y^{\prime}\right\|_{\Phi, s}=1$.

As we know $2 x_{0}=x_{n}^{\prime}+y_{n}^{\prime}$; then $k\left(2 x_{0}-y_{n}^{\prime}\right)-h y_{n}^{\prime} \stackrel{\mu}{\rightarrow} 0$. It implies that $k\left(2 x_{0}-y_{n}^{\prime}\right)-h y_{n}^{\prime} \stackrel{w^{*}}{\longrightarrow} 0$. Combining with the proof 
above $y_{n}^{\prime} \stackrel{w^{*}}{\longrightarrow} y^{\prime}$ and $\left\|y^{\prime}\right\|_{\Phi, s}=1$, we have $y^{\prime}=(2 k /(k+h)) x_{0}$. As a result, $2 k=k+h$. So $k=h$. We have $x_{n}^{\prime}-y_{n}^{\prime} \stackrel{\mu}{\rightarrow} 0$ as $n \longrightarrow \infty$. Namely,

$$
2\left(x_{n}^{\prime}-x_{0}\right)=x_{n}^{\prime}-y_{n}^{\prime} \stackrel{\mu}{\rightarrow} 0 \quad(n \longrightarrow \infty) .
$$

By the proof above, we get $1<k_{0}=k$; thus

$$
k_{n}^{\prime} x_{n}^{\prime}-k_{0} x_{0} \stackrel{\mu}{\rightarrow} 0 \quad(n \longrightarrow \infty) .
$$

Step 3. We will show that $I_{\Phi}\left(k_{n}^{\prime} x_{n}^{\prime}\right) \longrightarrow I_{\Phi}\left(k_{0} x_{0}\right)$. In fact

$$
\begin{aligned}
& s\left(I_{\Phi}\left(k_{0} x_{0}\right)\right)=k_{0}, \\
& s\left(I_{\Phi}\left(k_{n}^{\prime} x_{n}^{\prime}\right)\right) \longrightarrow k \quad(n \longrightarrow \infty),
\end{aligned}
$$

so $s\left(I_{\Phi}\left(k_{n}^{\prime} x_{n}^{\prime}\right)\right) \longrightarrow s\left(I_{\Phi}\left(k_{0} x_{0}\right)\right)(n \longrightarrow \infty)$. By the fact that $s(u)>1$ and $s(u)-1>0$, now $s(u)$ is strictly monotonous on $[u,+\infty)$. Hence, we have

$$
I_{\Phi}\left(k_{n}^{\prime} x_{n}^{\prime}\right) \longrightarrow I_{\Phi}\left(k_{0} x_{0}\right) \quad(n \longrightarrow \infty)
$$

Corollary 10. Let $s(u)>1$ with $u>0$ and $\Phi$ be an Orlicz function. $L_{\Phi, s}$ is middle point locally uniformly convex if and only if $\Phi \in \triangle_{2}$ and $L_{\Phi, s}$ is strictly convex.

\section{Data Availability}

No data were used to support this study.

\section{Conflicts of Interest}

The authors declare that they have no conflicts of interest.

\section{Acknowledgments}

Yunan Cui is supported by NSF of China (Grant no. 11871181).

\section{References}

[1] M. A. Rieffel, "Dentable subsets of Banach spaces, with applications to a Radon-Nikodym theorem," in Proceedings of the Conference of Functional Analysis, pp. 71-77, Washington, DC, USA, 1967.

[2] H. B. Maynard, "A geometrical characterization of Banach spaces with the Radon-Nikodym property," Transactions of the American Mathematical Society, vol. 185, pp. 493-500, 1973.

[3] W. J. Davis and R. R. Phelps, "The Radon-Nikodym property and dentable sets in Banach spaces," Proceedings of the American Mathematical Society, vol. 45, pp. 119-122, 1974.

[4] B.-L. Lin, P.-K. Lin, and S. L. Troyanski, "Characterizations of denting points," Proceedings of the American Mathematical Society, vol. 102, no. 3, pp. 526-528, 1988.

[5] S. Chen, "Geometry of Orlicz spaces," Dissertationes Mathematicae, 1996.
[6] J. Musielak, "Orlicz spaces and modular spaces," Lecture Notes in Mathematics, vol. 1034, no. 4, pp. 1-216, 1983.

[7] H. Hudzik and L. Maligranda, "Amemiya norm equals Orlicz norm in general," Indagationes Mathematicae, vol. 11, no. 4, pp. 573-585, 2000.

[8] Y. Cui, L. Duan, H. Hudzik, and M. Wisła, "Basic theory of $p$ Amemiya norm in Orlicz spaces $(1 \leq p \leq \infty)$ : extreme points and rotundity in Orlicz spaces endowed with these norms," Nonlinear Analysis, vol. 69, no. 5, pp. 1796-1816, 2008.

[9] M. Wisła, "Geometric properties of Orlicz spaces equipped with p-Amemiya norms - results and open questions," Commentationes Mathematicae, vol. 55, no. 2, pp. 183-209, 2015.

[10] Y. Cui, H. Hudzik, J. Li, and M. Wisła, "Strongly extreme points in Orlicz spaces equipped with the $p$-Amemiya norm," Nonlinear Analysis, vol. 71, no. 12, pp. 6343-6364, 2009.

[11] Y. Cui, H. Hudzik, and R. Płuciennik, "Extreme points and strongly extreme points in Orlicz spaces equipped with the Orlicz norm," Zeitschrift für Analysis und ihre Anwendungen Journal for Analysis and its Applications, vol. 22, no. 4, pp. 789817, 2003.

[12] H. Hudzik, W. Kurc, and M. Wisła, "Strongly extreme points in Orlicz function spaces," Journal of Mathematical Analysis and Applications, vol. 189, no. 3, pp. 651-670, 1995.

[13] R. Kaczmarek, "Uniform rotundity of Orlicz function spaces equipped with the $p$-Amemiya norm," Mathematische Nachrichten, vol. 291, no. 3, pp. 1514-1532, 2018.

[14] A. Kamińska, "Extreme points in Orlicz-Lorentz spaces," Archiv der Mathematik, vol. 55, no. 2, pp. 173-180, 1990.

[15] H. W. Milnes, "Convexity of Orlicz spaces," Pacific Journal of Mathematics, vol. 7, no. 3, pp. 1451-1483, 1957.

[16] B. Turett, "Rotundity of Orlicz Spaces," Indagationes Mathematicae, vol. 79, no. 5, pp. 462-469, 1976.

[17] P. Wang, F. Yu, and Y. Cui, "Strongly extreme points in MusielakOrlicz spaces with the Orlicz norm," Journal of Analysis and its Applications, vol. 28, no. 2, pp. 223-232, 2009. 


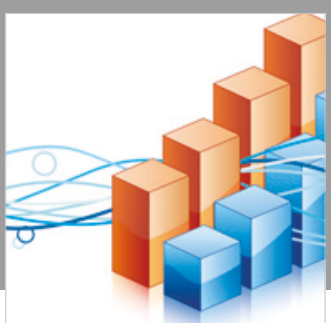

Advances in

Operations Research

\section{-n-m}
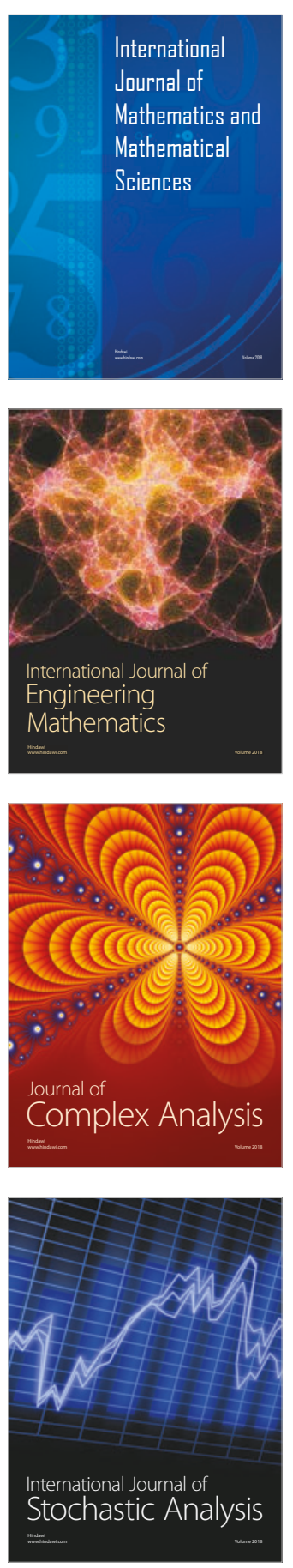
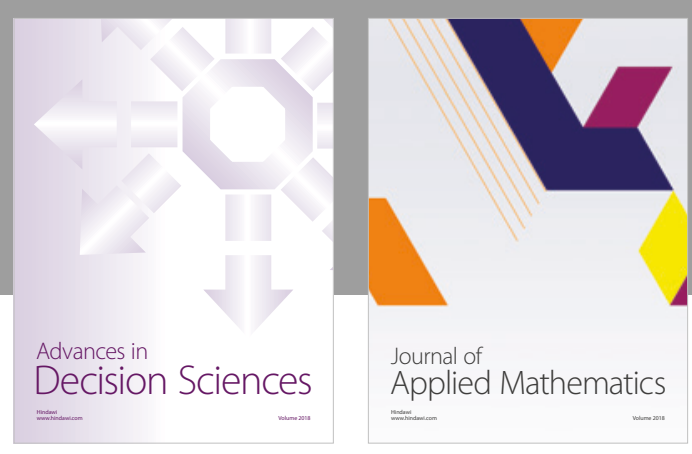

Journal of

Applied Mathematics
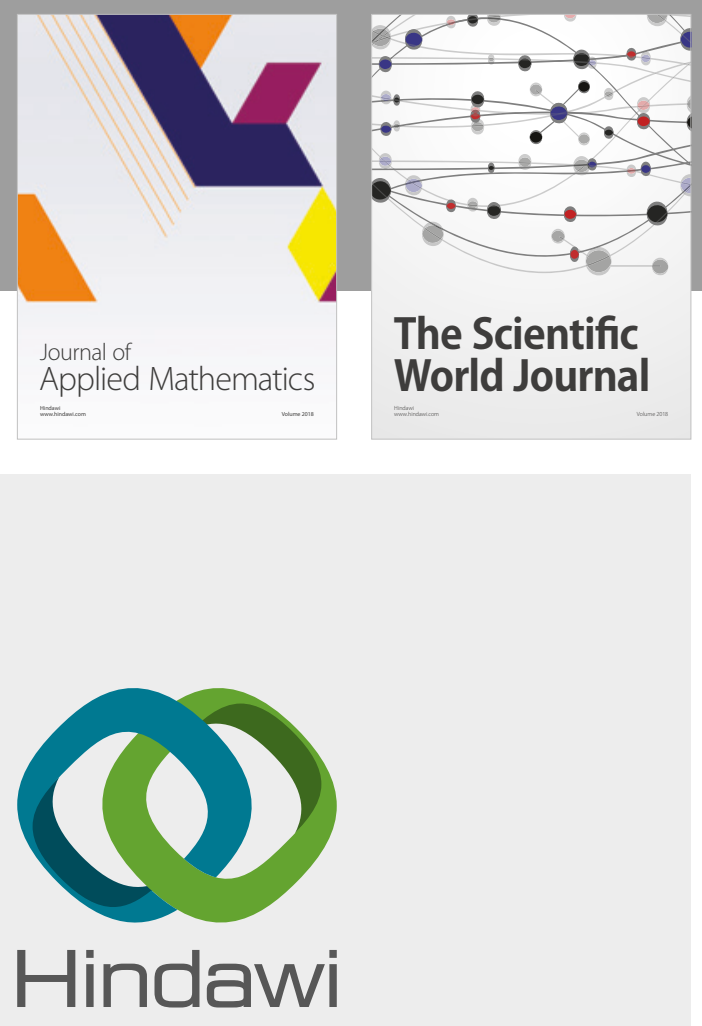

Submit your manuscripts at

www.hindawi.com

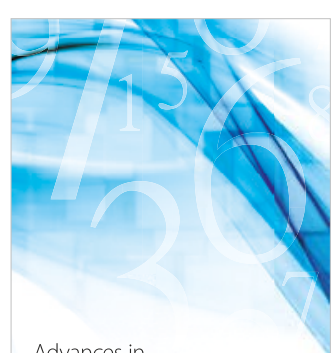

Advances in
Numerical Analysis
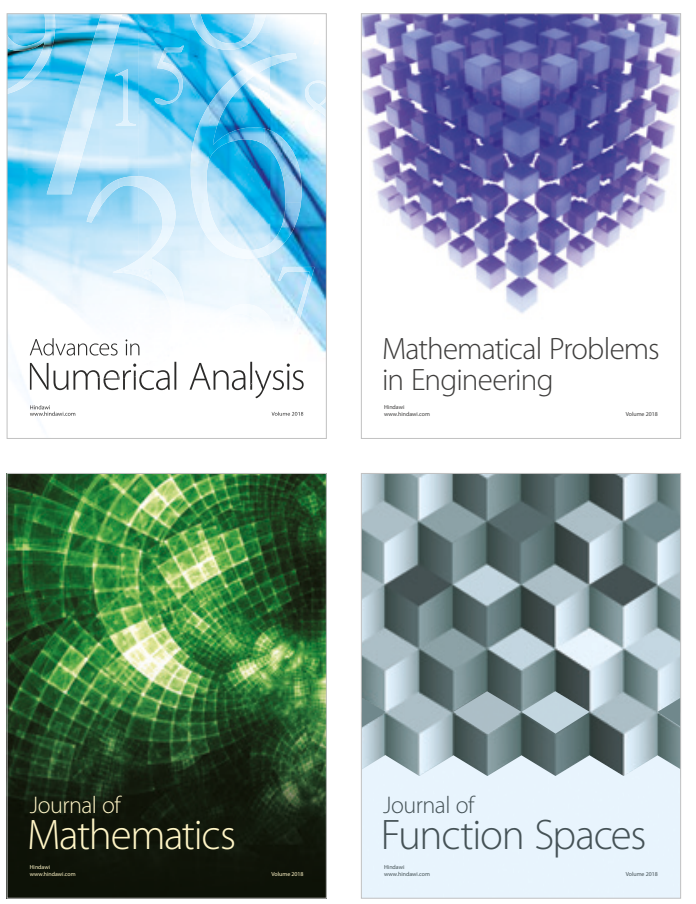

Mathematical Problems in Engineering

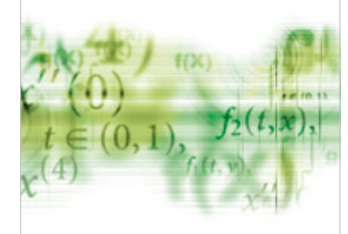

International Journal of

Differential Equations

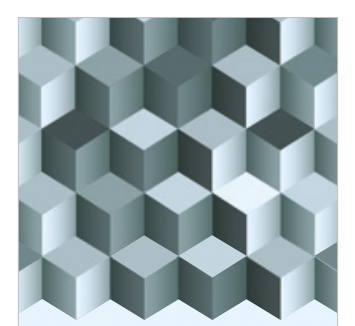

Journal of

Function Spaces

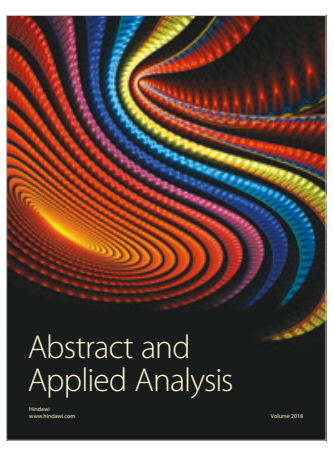

The Scientific

World Journal

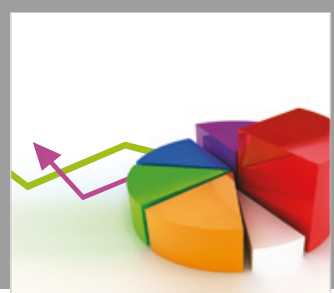

Journal of

Probability and Statistics
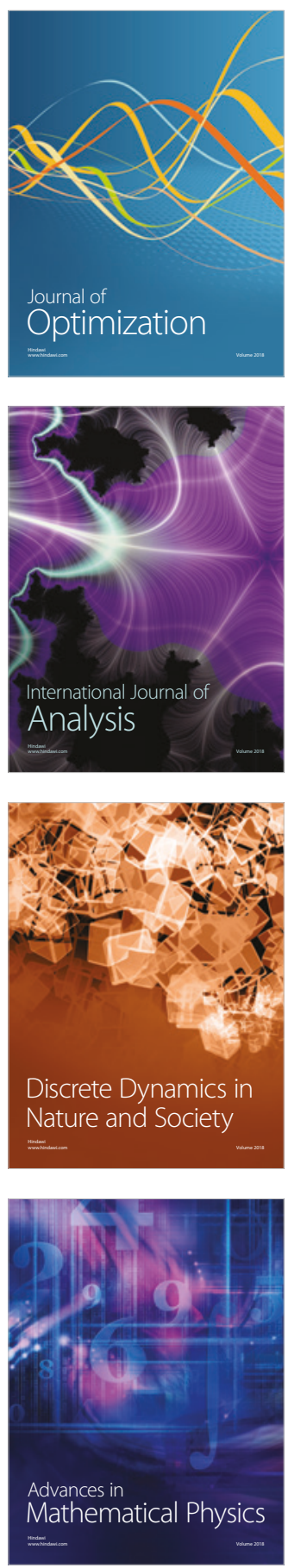